\title{
Ultraschallunterricht während des Medizinstudiums: Bilanz nach Ablauf eines Studienjahres in Lausanne, Schweiz
}

Die Medizinstudentinnen und -studenten der Universität Lausanne erwerben im Rahmen eines geblockten Radiologie Kurses im 4. Studienjahr (1. Jahr des Masterstudiums) erste Kenntnisse in der Sonografie. Während dieses einwöchigen Kurses nehmen die Studierenden in Gruppen von sieben bis zehn Personen an einem dreistündigen Ultraschallworkshop unter der Leitung erfahrener Tutorinnen und Tutoren teil. Im Sommersemester 2016 absolvierten $80 \%$ der Studierenden im ersten Jahr des Masterstudiums diese Blockveranstaltung, das sind 114 Studierende. Die restlichen Studierenden dieses Jahrgangs werden die Blockveranstaltung am Ende des Wintersemesters 2016/17 absolvieren.

Im Rahmen dieser dreistündigen Ultraschallworkshops, die in einem eigens dafür bereitgestellten Saal abgehalten werden ( $\vee$ Abb. 1), wird den Studierenden zuerst theoretisches Wissen vermittelt, das danach sofort in praktischen Übungen umgesetzt wird. Thema der Workshops ist unter anderem:

- die Einstellung des Ultraschallgeräts, sowie die Beschreibung der wichtigsten Einstellungen und Demonstration ihrer Auswirkungen
- die Bedienung und Auswahl von Ultraschallsonden

- die normale sonografische Anatomie des Abdomens und konventionelle

Abdomenultraschallbilder

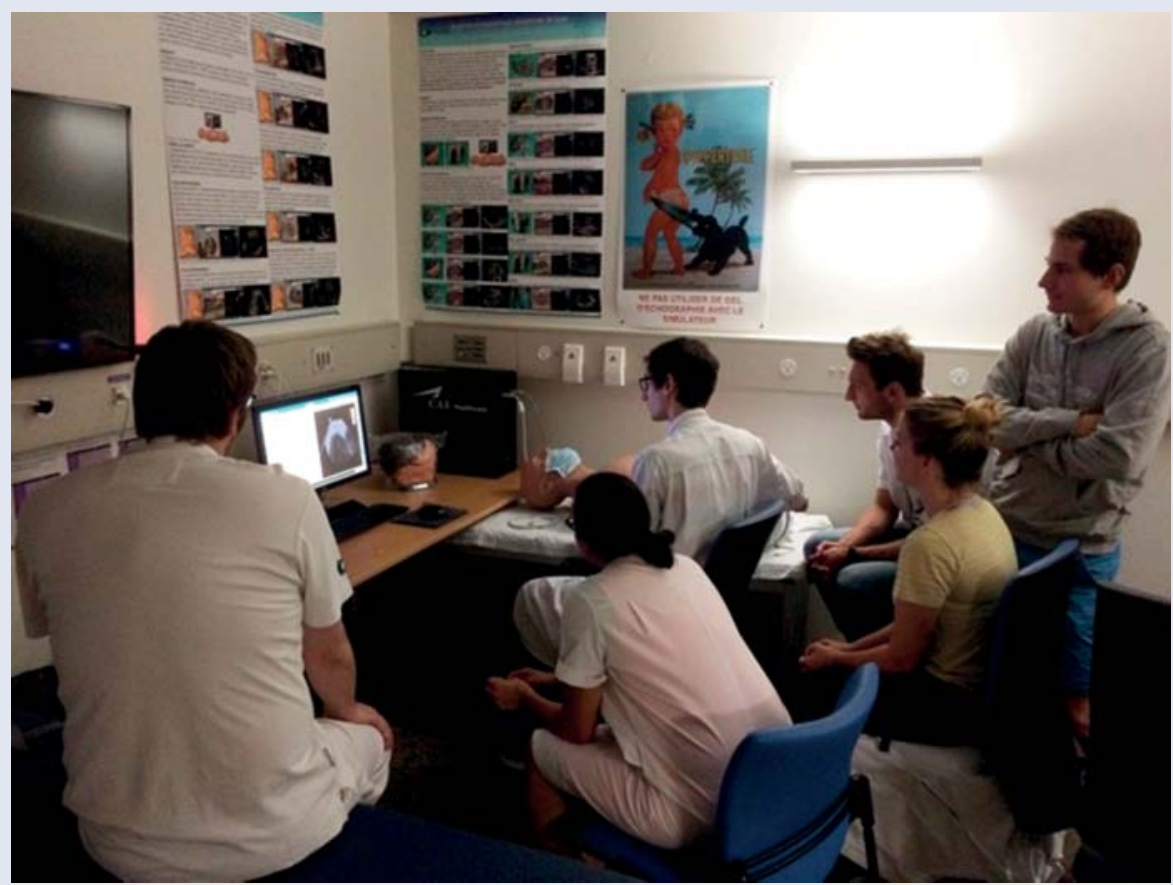

- Abb. 1 Die Studenten folgen einem ihrer Kollegen bei der Übung am Simulator. Der Tutor bleibt im Hintergrund.
- FAST/eFAST-Untersuchungen und die Suche nach freier Flüssigkeit mit einem Ultraschallsimulator 


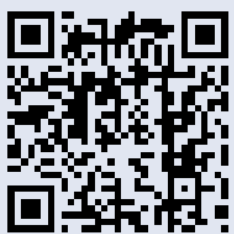

Abb. 2 Grundeinstellungen des Ultraschallgeräts.

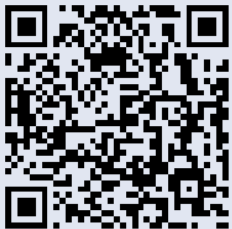

Abb. 3 Grundzüge der sonografischen Anatomie des Abdomens.

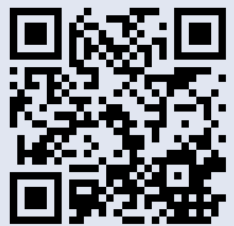

- Abb. 4 FAST eFAST Deutsch.

Die Einstellungen am Apparat, die normale Anatomie und das FAST/eFAST-Prinzip sind außerdem auf Plakaten, die im Saal angebracht sind, beschrieben. Die Tutorinnen und Tutoren können sich jederzeit auf diese Plakate beziehen. Gleichzeitig können die Studierenden auch während der Übungen auf die Plakate zurückgreifen. Die Plakate stehen auch als PDF auf der Website der Abteilung für Radiodiagnostik und interventionelle Radiologie des CHUV zum Download bereit. Verwenden Sie dafür bitte die QR-Codes am Ende dieses Artikels ( Abb. 2-4).

Die Studierenden erhalten außerdem ein Nachschlageheft, in dem die Einstellungen, die konventionellen Bilder der normalen Anatomie und die Technik zum Einsatz des FAST/eFAST-Prinzips noch einmal erklärt werden. In diesem Nachschlageheft finden sie auch die Normalwerte bei einer Reihe von sonografischen Messungen bei Erwachsenen und Kindern.

Nach dem theoretischen Unterrichtsteil, der jeweils 10 - 15 Minuten zu Beginn des Workshops dauert, werden die Studierenden zwei verschiedene Arbeitsplätze für die praktischen Übungen zugeteilt. Der erste Arbeitsplatz ist mit einem Ultraschallgerät zur Untersuchung am Phantom ausgestattet, der zweite mit einem Ultraschallsimulator. An beiden Arbeitsplätzen besteht das vorrangige Lernziel darin, die Sonde richtig zu halten und die Bilder korrekt auszurichten. Die Studierenden führen in der Folge mit Hilfe der Plakate oder des Nachschlageheftes konventionelle Bildgebungen für verschiedene abdominale Organe durch. Sobald die Studierenden diese Grundlagen beherrschen, verlangen sie meist sehr bald nach der Möglichkeit untereinander zu üben, unter Verwendung des Ultraschallgeräts. Sie haben außerdem die Möglichkeit, den Saal nach den Workshops für einige Stunden selbstständig zu benützen. Die Workshops werden mit FASTÜbungen am Simulator beendet. Am Simulator können positive FAST-Situationen programmiert werden, die die Studierenden dann blind einer Untersuchung unterziehen und feststellen müssen, ob sie positiv ist oder nicht. Auch hier können die Studierenden selbstständig mithilfe von Übung Codes, die zufällig ausgegeben werden, üben.

Der erste Jahrgang an Studierenden, die an dem geblockten Radiologiekurs teilnahmen, erhielt einen Feedbackbogen. Die Rücklaufquote betrug $91 \%$. Die Frage, ob Ultraschallunterricht in ihrem Studienabschnitt nützlich für sie sei, wurde einstimmig bejaht. In Bezug auf den Aufbau der Workshops gaben nur 25-28\% der Studierenden an, dass sie Themen bezüglich der Kenntnis der Geräte und ihrer Einstellungen als nützlich empfanden. Neunzehn bis $22 \%$ der Befragten fanden die Auswahl dieser Themengebiete hervorragend, was bedeutet, dass die Hälfte der Studierenden sie als entweder nur ausreichend oder sogar nutzlos betrachtete.

Das Studium der normalen Anatomie wurde von $89 \%$ der Befragten als gut oder sogar hervorragend bezeichnet. Der Großteil der Studierenden befürwortete die Arbeit am Phantom oder am Simulator. Nur $11 \%$ (Simulator) bzw. 14\% (Phantom) bezeichneten diesen Aspekt der Ausbildung als ausreichend oder nutzlos.

Die Verwendung des Nachschlageheftes stieß auf gemischte Reaktionen: $35 \%$ der Befragten empfanden es als nützlich, $15 \%$ als gut und $20 \%$ als sehr nützlich.
Das Hauptziel dieser Workshops war es, am Ende des Kurses eine FAST/eFAST-Untersuchung durchführen zu können. Am dritten Tag hatten alle Studierenden die Möglichkeit, in Anwesenheit eines Tutors bzw. einer Tutorin, eine Übung auf dem Simulator durchzuführen.

Die Studierenden mussten dabei eine Situation blind evaluieren, die der Tutor oder die Tutorin zuvor am Simulator programmiert hatte. Die Umsetzung der Untersuchung und ihr Resultat (Vorfinden von freier Flüssigkeit oder nicht) wurden bewertet. Die Studierenden erhielten sofort ein konstruktives Feedback. Sämtlichen Studierenden gelang es (mit mehr oder weniger Geschick), die Untersuchung durchzuführen und die freie Flüssigkeit an den richtigen Stellen aufzufinden. Nichtsdestoweniger wurde deutlich kommuniziert, dass es nicht Ziel dieser Workshops war, eine Ausbildung in abdominalen UItraschalluntersuchungen zu vermitteln, da hierfür eine viel fundiertere Ausbildung notwendig ist.

Auf dem Feedbackbogen gaben $79 \%$ der Studierenden an, dass sie sich sicher im Umgang mit FAST/eFAST fühlten. Diese Zahl spiegelt auch den Eindruck vonseiten der Tutorinnen und Tutoren nach Abhaltung der FAST-Übungen wider. Im Gegensatz dazu gaben nur 47 \% der Studierenden an, dass sie sich nicht in der Lage fühlten, eine abdominale Ultraschalluntersuchung durchzuführen, trotz des deutlichen Hinweises der Tutorinnen und Tutoren, dass das Ziel der Workshops auf die Vermittlung der FAST-Untersuchung beschränkt sei.

Die Tatsache, dass sich $53 \%$ der Studierenden dazu befähigt fühlen, eine Ultraschalluntersuchung des Bauchraums durchzuführen, sollte uns dazu Anlass geben, über unsere Rolle als Lehrende im Bereich der Sonografie nachzudenken. Immer mehr Vertreter des Gesundheitswesens stellen den Anspruch, Ultraschalluntersuchungen durchzuführen. Wenn sich Studierende der Medizin bereits nach drei Stunden Unterricht und praktischen Übungen dazu befähigt fühlen, selbstständig abdominale Ultraschalluntersuchungen durchzuführen, werden andere Dienstleister im Gesundheitsbereich wohl eine ähnliche 
Einstellung haben. Deswegen ist es unabdinglich, den Eindruck der Studierenden, eine solche Untersuchung sei einfach und unkompliziert, bereits jetzt zu berichtigen.

Beinahe die Hälfte der Studierenden versteht, dass es sich bei der abdominalen Sonografie um ein komplexes diagnostisches Verfahren handelt. Indem man diese Botschaft im Zuge der Praktika immer wieder vermittelt und den Studierenden eventuell auch andere Ausbildungen im Bereich Ultraschall nahelegt, sollte es möglich sein, allen die Komplexität dieses Verfahrens und die Schwierigkeit der Ausbildung verständlich zu machen. Wenn junge Medizinerinnen und Mediziner sich bereits während des Studiums grundlegende Kenntnisse der sonografischen Technik aneignen und die Komplexität dieser Untersuchung verstehen, sind sie sicherlich selbstkritischer, sobald sie im
Zuge ihrer postgradualen Ausbildung selbst die Ultraschallsonde in die Hand nehmen müssen.

Mit Sicherheit steht folgendes fest: Die Studierenden dieses Jahrgangs waren einhellig begeistert von dem Unterricht in gezielten Ultraschalluntersuchungen und wissen nun, dass ein Ultraschallgerät eingeschaltet sein muss um zu funktionieren, dass das Bild nicht wie von Zauberhand auf dem Bildschirm erscheint und man die Einstellungen des Geräts beachten muss, dass die Sonde nicht immer ein schönes Bild produziert, dass man Ultraschallgel verwenden muss und, nicht zuletzt, dass es polytraumatisierten Patientinnen und Patienten das Leben retten kann, wenn man freie Flüssigkeit findet. Kenntnisse im Basic Life Support (BLS) werden in den verschiedensten Kursen auf sämtlichen Niveaus vermittelt. Nun müssen die Studie- renden der Medizin weiterhin in der FASTTechnik unterrichtet werden.

Die Unterrichtsplakate stehen auf Deutsch und Französisch im PDF-Format auf der Website der Abteilung für Radiodiagnostik und interventionelle Radiologie des Universitätsspitals Lausanne zum Download bereit.

Um zu den PDF-Dokumenten auf Deutsch zu gelangen, scannen Sie bitte die QRCodes.

Prof. Dr. med. Jean-Yves Meuwly Abteilung für diagnostische und interventionelle Radiologie Universitätsspital Lausanne Schweiz 